\title{
Comparison between patients' and parents' satisfaction level towards cleft management using Cleft Evaluation Profile
}

\author{
Lau Kim Lian ${ }^{1 *}$, Ong Siang Ching1, Wan Salina Wan Sulaiman² \\ ${ }^{1}$ Unit Ortodontik, Klinik Pergigian Kota Bharu, Jalan Mahmood, 15200 Kota Bharu, Kelantan, Malaysia. \\ 2 Pejabat Timbalan Pergigian Kesihatan Negeri (Pergigian) Kelantan, Aras 7, Bangunan KWSP, Jalan Padang \\ Garong, 15598 Kota Bharu, Kelantan, Malaysia.
}

\begin{abstract}
Cleft lip and palate (CLP) are common birth defects that occur in approximately 1 out of every 611 newborn in Malaysia. Cleft Evaluation Profile (CEP) is a well-established method used to evaluate the patient's level of satisfaction with the CLP treatment that they received. This study aimed to compare the level of satisfaction of cleft patients and their parents towards cleft management in Kelantan, Malaysia. The results showed that the mean scores of CEP for the parents were between 2.04 and 3.00, whilst the patient's mean score ranged from 2.01 to 3.04. In general, nose is the most dissatisfied feature for both patients and parents with the mean score of 3.04 for patients and 3.00 for parents. Teeth was the second highest (2.97) mean scores rated by parents but it was the third highest (2.84) mean score from the patients. Lip feature scored the third highest mean score for parents, and it was the second-highest mean score for patients. Hearing was the lowest mean score for both parents and patients. Both parties were aware of the features of their facial and nose appearance. From the weighted Kappa statistic, both the parent and patient pairs demonstrated good to very good agreement for their satisfaction towards the clinical outcome. In conclusion, features related to facial appearance (nose, teeth, lip and facial profiles) require more attention and improvement during the treatment process.
\end{abstract}

Keywords: cleft lip and/ or palate (CLP), level of satisfaction, Cleft Evaluation Profile (CEP)

\section{Introduction}

Cleft lip and/or palate (CLP) are common facial birth defects that occur among the children and adults (Noor et al., 2007). The occurrence of CLP was reported to be approximately 1 in 700 live births in Europe (Ali \& Sahito, 2015). Meanwhile, 1 in 611 children in Malaysia was born with CLP in 2007 (NOHSS, 2007). A recent study revealed that Asians are more commonly
Received:

15 December 2020

Revised:

25 January 2021

Accepted:

3 February 2021

Published Online:

28 February 2021

\section{How to cite this article:}

Lau, K. L., Ong, S. C., \& Wan Sulaiman, W. S. . Comparison between parents' and patients' satisfaction level towards cleft management using Cleft Evaluation Profile. IIUM Journal of Orofacial and Health Sciences, 2(1)., $\quad 37-45$. https://doi.org/10.31436/ijoh s.v2i1.63

\section{Article DOI:}

https://doi.org/10.31436/ijohs. $\underline{\mathrm{v} 2 \mathrm{i} 1.63}$

Corresponding author:

Address:

Unit Ortodontik, Klinik Pergigian Kota Bharu, Jalan Mahmood, 15200 Kota Bharu, Kelantan, Malaysia.

Telephone: +609- 7484084

Fax: +609- 7484157

Email address:

laukimlian1012@gmail.com affected with this cranio-facial abnormality compared to Caucasians (Natsume \& Kawai, 1986).

Previous study in Malaysia found that the incidence of oral cleft alone was higher in females (56.7\%) than males $(43.3 \%)$ (Ali \& Sahito, 2015). On the other hand, from a recent study in Italy revealed that CLP was found significantly more common in males (67.6\%) than females (Gatti et al., 2017). 
CLP patients generally require a multidisciplinary team management approach, a proper set of diagnosis, and stage-wise treatment planning protocols to identify and manage a full spectrum of complexities associated with the congenital deformities. This service of multidisciplinary care should start from infancy and continues into early adulthood as long-term treatment planning with proper standardized surgical protocols can improve the overall desired quality of esthetic and functional treatment outcome significantly (Oosterkamp et al., 2007).

For the past two decades, numerous studies have been conducted to assess the level of satisfaction of individuals with CLP and their parents concerning the esthetics and speech features in cleft management and treatment outcomes (Luyten et al., 2013; Noor et al., 2007 \& Oosterkamp et al., 2007). From earlier studies, a high level of satisfaction in cleft care and surgical outcomes was observed from the CLP patients and their parents and they were less satisfied with the cleft related subunit in the nasolabial region, particularly teeth (Luyten et al., 2013). On the other hand, a disappointing level of satisfaction from CLP patient and their parents was confirmed from a survey from the Clinical Standards Advisory Group investigation (CSAG) in the United Kingdom (Williams et al., 1993). Twenty years later, after centralization of the cleft care with regards to CSAG in UK, another similar study was conducted and it showed that there was improvement in overall patient and parental satisfaction levels (Sell et al., 2015).

In a recent study, significant differences in the levels of satisfaction were found between the parents from Asian and western countries. Chinese parents from China generally shows a higher level of satisfaction level in the lip and facial profile and a lower level of satisfaction level in the nose and teeth features (Ha et al., 2016). Another local study from Universiti Sains Malaysia Hospital (HUSM) revealed a higher parental satisfaction level in hearing and lower satisfaction level in teeth, lips and nose features (Noor et al., 2007). On the other hand, Italian parents were satisfied with the surgical outcomes in the nose and occlusal features, but were less dissatisfied with the breathing (Gatti et al., 2017). Ugandan parents were satisfied with the hearing, face and lip and less satisfied with the speech, teeth, and nose (Luyten et al., 2013).

To date, there is no study to compare the level of satisfaction between patients and parents towards cleft management in the CCC that was established over the past nineteen years. This study aimed to compare the level of satisfaction of cleft patients and their parents towards cleft management in Kelantan, Malaysia.

\section{Methods}

\section{Study participants}

This observational research study involved CLP patients and their parents who visited the Orthodontic Unit in the Combined Cleft Clinic (CCC), Kota Bharu, Kelantan from January 2017 to September 2017. Under a confidence level of 95\%, with study power set to $80 \%$, and alpha value of $5 \%$, a sample size of 27 patient-parent pairs was needed for this study. By considering the 10\% drop out cases, the final sample size of this study was 30 patient-parent pairs. The age range for patients was 17 to 25 years, whilst for parents was 18 to 65 years. The mean age of patients and parents was 17.2 and 39.8, respectively. Subjects with craniofacial syndrome, hearing or neurological impairment, and intellectual disabilities as well as subjects who were unable to answer the questionnaire were excluded from the study. The cleft patients in this study are still undergoing treatment and review appointments. $100 \%$ response rate was attained.

Ethical approval was obtained by following the guidelines stated in National Institutes of Health (NIH) under the Ministry of Health (MOH) Malaysia (NMRR-15-2497-28426). The ethical review was appraised and approved by the NIH Research Review Panel (JPP-NIH) and Medical Research and Ethics Committee (MREC) prior to data collection. 


\section{Semi-structured questionnaires}

Semi-structured self-administrated questionnaires were adopted from previous work of (Noor et al., 2007). Both English and Malay language versions of the questionnaire were made available for the correspondents. One of the parents who attended the CCC along with the CLP patients was asked to answer the parent questionnaire. The cleft patients were answered the CEP themselves. Full confidentiality for every subject was assured, by emphasizing and making clear that the participation in this study was not related to the child cleft care management before parent started answering the questionnaire. Informed consent was taken from parents (Questionnaire \& CEP) and the CLP patient (CEP). Before proceeding to the more specific questions related to the generic information, the questionnaire was designed to make the participants to be more comfortable by starting with a set of identical questions related to superfluous topics, for example, "How long does it take for you to travel from home to clinic?". The overall satisfaction towards cleft management was evaluated by using forced response questions, including:

"Overall how satisfied are you with the care and attention that you have received from the cleft team?"

$\begin{array}{ll}\text { very satisfied } & \text { (CODED) } \\ \text { satisfied } & \text { (CODED) } \\ \text { reasonably satisfied } & \text { (CODED) } \\ \text { dissatisfied } & \text { (CODED) } \\ \text { very dissatisfied } & \text { (CODED) }\end{array}$

\section{Cleft Evaluation Profile}

The Cleft Evaluation Profile (CEP) is a wellestablished approach originating from the Royal College of Surgeons Cleft Lip and Palate Audit Group, used to investigate the level of satisfaction for individual features in the cleft management (Turner et al.,1997). There are eight individual items within the CEP including speech, hearing, appearance of teeth, appearance of lip, appearance of nose, breathing through the nose, profile of the face, and bite. Each item was paired with a 7-point Likert scale, where the subjects were requested to rate their level of satisfaction from very satisfactory (a rank of 1) to very unsatisfactory (a rank of 7) (Table 1). Besides, it was a valid and valuable assessment tool to evaluate cleft patients' level of satisfaction for the treatment received (Noor et al., 2007). In this study, the CEP was distributed to both parents and patients to evaluate their level of satisfaction with regards to the quality of cleft treatment provided.

\section{Statistical analysis}

Data was analysed by using Statistical Package for Social Sciences (SPSS) software program version 18.0 (IBM Corporation, Armonk, NY, USA). The weighted kappa statistic was used to assess the degree of agreement in the level of satisfaction perceived between parent and patient pairs regarding the CEP for the cleft management outcome. The evaluation method used for the analysis of the level of agreement was adopted from the recommended classification by Altman (1999) (Table 2).

\section{Results}

Out of the total 69 parents and 69 patients, 14 parents and 23 patients were male, whilst 55 parents and 46 patients were female. Vast majority of our subjects were Malay $(n=65$; $94.2 \%)$, followed by 3 Chinese $(4.3 \%$ ) and 1 Indian (1.4\%). The mean age of the parents and patient groups were 39.8 and 17.2, respectively. The sociodemographic and economic characteristics of the subjects were summarized in Table 3 and 4.

Two-thirds of the parents attended only primary school (n=8; 11.6\%) and secondary school $(n=39,56.5 \%)$. More than half of them had gross household income of less than RM $2999(\mathrm{n}=45,65.2 \%)$ per month. Most of the parents were working in private sectors $(\mathrm{n}=33,47.8 \%)$, followed by government servants $(n=29,42.0 \%)$ and labourers $(n=7$, $10.2 \%)$. 
Table 1. Cleft Evaluation Profile Please circle the number that is closet to how things are for you (patient)/ your child (parent) now.

\section{A. Speech}

Very satisfactory

12

3

\section{B. Hearing}

Very satisfactory

12

2

3

4

C. Appearance of the teeth Very satisfactory

12

D. Appearance of the lip

Very satisfactory

$1 \quad 2$

E. Appearance of the nose

Very satisfactory

123

4

F. Breathing through the nose Very satisfactory

$\begin{array}{llll}1 & 2 & 3 & 4\end{array}$

G. Profile of the face Very satisfactory

1

2

3
Very unsatisfactory

$\begin{array}{lll}5 & 6 & 7\end{array}$

Very unsatisfactory

$\begin{array}{lll}5 & 6 & 7\end{array}$

Very unsatisfactory

$5 \quad 6 \quad 7$

D.

Very unsatisfactory

$5 \quad 6 \quad 7$

E.

Very unsatisfactory

$5 \quad 6 \quad 7$

Very unsatisfactory

$5 \quad 6 \quad 7$

G.

Very unsatisfactory

$5 \quad 6 \quad 7$

Very unsatisfactory

$\begin{array}{lll}5 & 6 & 7\end{array}$

B.

C.

F.

H.

A.

.

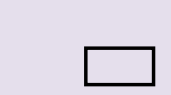

\section{H. Bite}

Very satisfactory

1

2

3

4

Table 2. Interpretation of strength of agreement for the kappa statistic

$\begin{array}{ll}\text { Value of kappa (k) } & \text { Strength of agreement } \\ <0.20 & \text { Poor } \\ 0.21-0.40 & \text { Fair } \\ 0.41-0.60 & \text { Moderate } \\ 0.61-0.80 & \text { Good } \\ 0.81-1.00 & \text { Very good }\end{array}$

Table 3. Sociodemographic characteristics of parents and patients

$\begin{array}{lll}\text { Variables } & \begin{array}{l}\text { No. of parents } \\ \text { n (\%) }\end{array} & \begin{array}{l}\text { No. of patients } \\ \text { n (\%) }\end{array} \\ \text { Sex } & 14(20.3) & 23(33.3) \\ \text { Male } & 55(79.7) & 46(66.6) \\ \text { Female } & & \\ \text { Ethnicity } & 65(94.2) & 65(94.2) \\ \text { Malay } & 3(4.3) & 3(4.3) \\ \text { Chinese } & 1(1.4) & 1(1.4) \\ \text { Indian } & \end{array}$


Table 4. Sociodemographic and economic characteristics of parents

$\begin{array}{ll}\text { Variables } & \text { n (\%) } \\ \text { Level of education } & \\ \text { Primary school } & 8(11.6) \\ \text { Secondary school } & 39(56.5) \\ \text { College/ University } & 22(31.9) \\ \text { Gross household income per month } & \\ \text { < RM 1000 } & \\ \text { RM 1000-2999 } & 21(30.4) \\ \text { RM 3000-4999 } & 24(34.8) \\ \text { RM 5000-9999 } & 9(13.1) \\ \text { > RM 10000 } & 12(17.4) \\ \text { Employment } & 3(4.3) \\ \text { Government servant } & \\ \text { Private sector } & 29(42.0) \\ \text { Laborer } & 33(47.8) \\ * \text { RM - Ringgit Malaysia, the Malaysian currency } & 7(10.2)\end{array}$

\section{Interaction with cleft team}

More than half of the parents felt at ease (64.2\%) when attending CCC. About onefourth felt very relaxed (25.9\%) and only $3.1 \%$ felt nervous when attending the clinic. Nearly half of the parents $(48.8 \%)$ preferred to discuss the child's treatment with the whole team together, whilst only one-third (35.8\%) preferred to meet each specialist separately.

\section{Families' contributions to decisions involving treatment}

More than half of the parents (58.7\%) were very much involved in making decisions about their child's treatment and $32.1 \%$ of them felt they were usually involved in their child's treatment. Only $9.2 \%$ felt that they were occasionally involved in the decision making.

\section{Overall satisfaction with cleft care}

Half of the parents (50.7\%) were very pleased with the operation outcome whilst about one-quarter of them were pleased $(26.1 \%)$ with the outcome and another 23.2 $\%$ were just satisfied. More than half $(59.4 \%)$ of the parents were very pleased with the cleft care services from the specialists, 36.2\% were satisfied, $3.0 \%$ were least satisfied and the remaining $(1.4 \%)$ were not satisfied. Parent's satisfaction level towards cleft management in Kelantan were summarized in Table 5 .

\section{Cleft Evaluation Profile}

The mean scores of CEP for the parents were between 2.04 and 3.00, whilst the patient's mean score ranged from 2.01 to 3.04. Nose feature has the highest mean score item of CEP for parents (3.00) and patients (3.04). Teeth was the second highest (2.97) mean scores rated by parents but it was the third highest (2.84) mean score from the patients. Lip feature scored the third highest mean score for parents, and it was the secondhighest mean score for patients. Hearing was the lowest mean score for both parents and patients. The results were shown in Figure 1.

The level of agreement between parents and patients for the satisfaction with the clinical outcomes was ranged from good to very $\operatorname{good}(\mathrm{k}=0.825, \mathrm{p}=0.000)$. Speech has a very good level of agreement while the other items in CEP scored a good level of agreement $(\mathrm{k}=0.691-0.778)$ (Table 6). 
Table 5. Level of parents' satisfaction towards cleft management in Kelantan.

\begin{tabular}{|c|c|c|}
\hline Variables & Variables & n (\%) \\
\hline Parents' feeling when attending the clinic & $\begin{array}{l}\text { Very relaxed } \\
\text { At ease } \\
\text { Reasonably at ease } \\
\text { Nervous }\end{array}$ & $\begin{array}{l}18(25.9) \\
44(64.2) \\
5(6.8) \\
2(3.1)\end{array}$ \\
\hline $\begin{array}{l}\text { Parents' involvement in making decisions } \\
\text { about child's treatment }\end{array}$ & $\begin{array}{l}\text { Very involved } \\
\text { Usually involved } \\
\text { Occasionally involved }\end{array}$ & $\begin{array}{l}41(58.7) \\
22(32.1) \\
6(9.2)\end{array}$ \\
\hline $\begin{array}{l}\text { Parents' preference to discuss child's } \\
\text { treatment with the whole team or with each } \\
\text { specialist separately }\end{array}$ & $\begin{array}{l}\text { Whole team together } \\
\text { Each specialist } \\
\text { separately } \\
\text { Don't know } \\
\text { Don't mind }\end{array}$ & $\begin{array}{l}34(48.8) \\
25(35.8) \\
4(6.1) \\
6(9.2)\end{array}$ \\
\hline $\begin{array}{l}\text { Parents' feeling about the outcome of } \\
\text { operation }\end{array}$ & $\begin{array}{l}\text { Very pleased } \\
\text { Pleased } \\
\text { Satisfied }\end{array}$ & $\begin{array}{l}35(50.7) \\
18(26.1) \\
16(23.2)\end{array}$ \\
\hline $\begin{array}{l}\text { Parents' feeling about the care and attention } \\
\text { received from specialists }\end{array}$ & $\begin{array}{l}\text { Very pleased } \\
\text { Satisfied } \\
\text { Least satisfied } \\
\text { Not satisfied }\end{array}$ & $\begin{array}{l}41(59.4) \\
25(36.2) \\
2(3.0) \\
1(1.4)\end{array}$ \\
\hline $\begin{array}{l}\text { Parents' feeling about the results and } \\
\text { outcome of treatment given by specialists }\end{array}$ & $\begin{array}{l}\text { Very satisfied } \\
\text { Satisfied } \\
\text { Least satisfied } \\
\text { Very not satisfied }\end{array}$ & $\begin{array}{l}36(52.2) \\
30(43.5) \\
2(2.9) \\
1(1.4)\end{array}$ \\
\hline
\end{tabular}

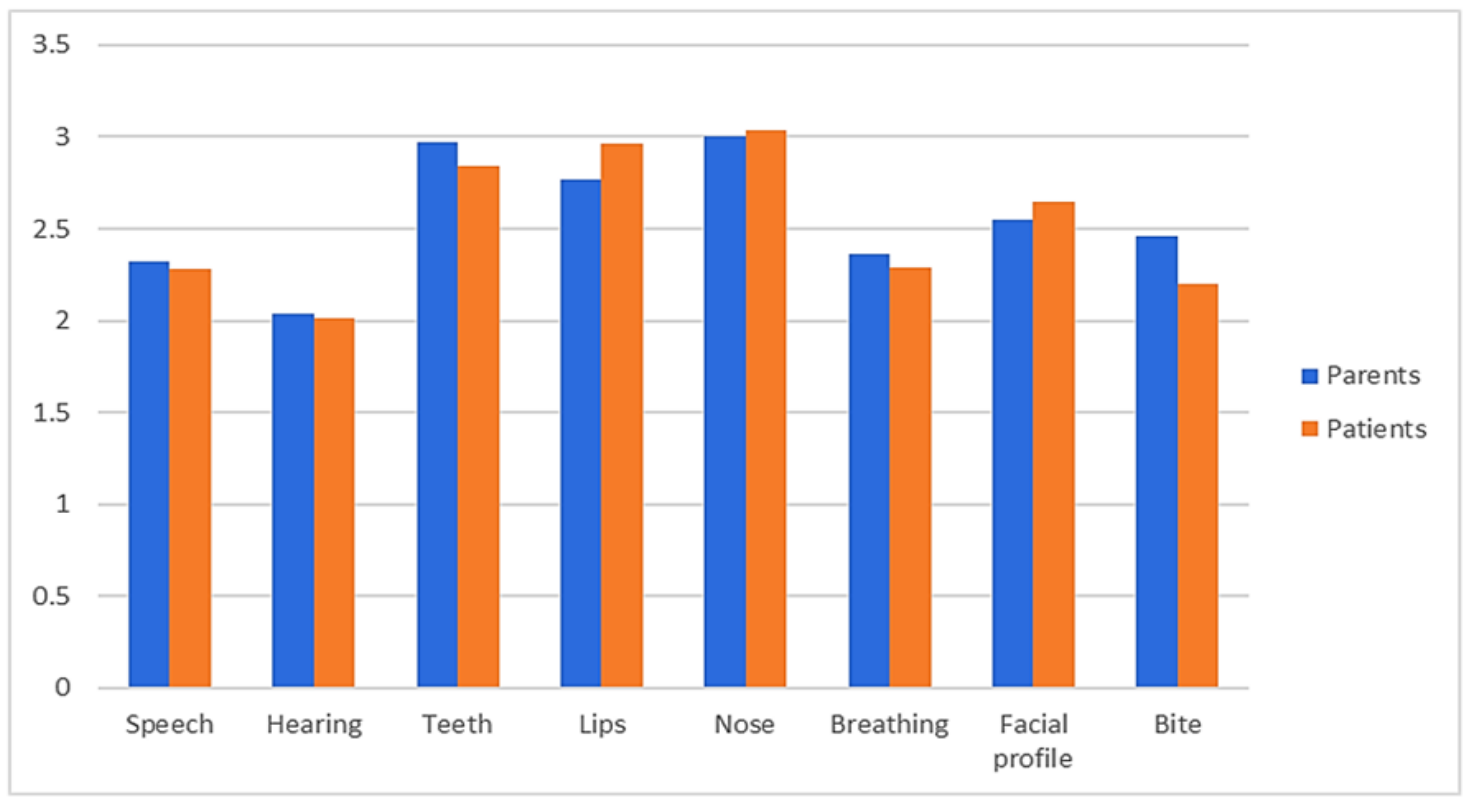

Figure 1. Mean scores of the Cleft Evaluation Profile for the parents and patients 
Table 6. Level of agreement between parents and patients for perceived satisfaction with clinical outcome

\begin{tabular}{lllll} 
Features & Kappa Value (k) & $\begin{array}{l}\text { Standard Error } \\
\text { (kappa) }\end{array}$ & Probability (p) & $\begin{array}{l}\text { Level of } \\
\text { agreement }\end{array}$ \\
\hline Speech & 0.825 & 0.054 & 0.000 & Very good \\
Hearing & 0.778 & 0.064 & 0.000 & Good \\
Teeth & 0.713 & 0.062 & 0.000 & Good \\
Lip & 0.691 & 0.065 & 0.000 & Good \\
Nose & 0.699 & 0.063 & 0.000 & Good \\
Breathing & 0.711 & 0.064 & 0.000 & Good \\
Face & 0.741 & 0.060 & 0.000 & Good \\
Bite & 0.750 & 0.061 & 0.000 & Good
\end{tabular}

\section{Discussion}

In this study, the Combined Cleft Clinic (CCC) located at the Main Government Dental Clinic in Kelantan is a referral centre for all cleft cases from all the districts in Kelantan (a northeast state at Peninsular Malaysia). This was established in 1998 and the average number of cleft patients received is about one hundred and fifty per year. The cleft management team consists of plastic surgeons, orthodontists, oral surgeons, dental paediatrician, and speech therapists. The clinical consultation is carried out twice a month on every first and third Tuesday afternoon. However, no study was conducted to compare the level of satisfaction between patients and parents towards cleft management in the CCC that was established over the past nineteen years.

Majority of the parents participated in this study were mother, consistent with another finding from China (Ha et al., 2016). Most of our participants in this study were from a lower socioeconomic status as found in another local study (Noor et al., 2007).

\section{Interaction with cleft team}

In our study, majority of the parents felt "at ease" when attending the clinic. This finding was in good agreement with a local study (Noor et al., 2007) in which about $68 \%$ of the parents felt "at ease" when they attended the clinic. The main reason is there was a specially allocated clinic for the cleft patients, where in the afternoon only cleft patients been seen. Cleft patients and their parents will be seated in the same waiting room. Thus, when sitting in the waiting room, there was no feeling of intimidation since every patient was having similar deformity. In addition, the cleft patient's parents even can share their experience along the journey of having cleft baby or cleft children.

\section{Families' contributions to decisions involving treatment}

More than half of the parents were very much involved in making decision, the findings were contradicted with the previous study (Noor et al., 2007). The main reason could be parents were given the clear comprehensive explanation of the cleft management during the CCC.

Effective communication between specialists and parents is crucial to omit the barrier of parents' involvement during the clinical discussion. About half of the group in our study were benefited by discussions of their child's treatment together with the whole cleft care team together. Our findings were consistent with the conclusions of a recent study in the United Kingdom, in which a centralised cleft management services improved the overall outcomes significantly compared to that of uncentralised service 15 years ago (Sell et al., 2015). In contrast, onethird of the parents preferred to have a clinical session with their specialist separately. They claimed that it would be more comfortable for them to discuss their problem and treatment plans in detail. A similar finding was also presented in a local study, in which parents were able to focus on one issue at a time and easier for them to appreciate more on their child's conditions (Noor et al., 2007). 


\section{Overall satisfaction with cleft care}

All parents were satisfied with the surgical outcome. Generally more than half of the participants were very satisfied with the care and attention received from the specialists. The reason why patients and parents were satisfied with the overall cleft care at our clinic could be due to the centralisation of cleft services into two hospitals in Kelantan. In that case, surgeons had enough number of cases to practice and to master their skills. Our findings were supported by a recent UK study, in which improved treatment outcomes were obtained after the implementation of cleft care centralisation (Sell et al., 2015).

\section{Cleft Evaluation Profile}

In our study, parents and patients gave more attention to the features related to facial appearance - nose, teeth, lip, mastication, and facial profile. These findings were in good agreement with Noar et al., (1991). The findings in our study showed "good to very good" satisfaction agreement which was in contrast to previous local study (Noor et al., 2007). This could be explained by the fact that our cleft care team consists of a multidisciplinary speciality including orthodontists, oral surgeon, plastics surgeon, paediatric dentistry specialists, and speech therapists. Thus, a closer monitoring and consistent follow up might contribute to a more satisfying treatment outcome. Analogous to Noor et al., (1998), both parents and patients were very dissatisfied on the nose feature because most patients are yet to undergo rhinoplasty surgery (mean age is 16.2 years old). The counselling session might help reduce parents' worry and should be conducted with the whole family (Lansdown et al., 1991).

The sample that we collected was from those who has not completed the treatment, so it does not reflect the overall level of satisfaction. For example, cleft patients were concerned about the nose but rhinoplasty was not yet done to correct the deformity. The level of satisfaction that we were assessing was derived from the group of patients that are still undergoing treatment. Thus, that is not a true level satisfaction for the overall cleft care. On the other hand, future study might be needed to investigate the dissatisfaction sources of the patients and parents in detail. To review the treatment outcomes and cleft care objectively, a more systematic protocol should be implemented to measure the appearance, dento-alveolar arch relationships, oral health, hearing, speech, and psychological aspects. It would be best if the clinical audits can be conducted locally by providing proper training in analysing personal practice (Sell et al., 2015).

A similar study should be conducted on patients who have undergone completed overall cleft treatment including orthognathic surgery and rhinoplasty to assess the overall level of satisfaction perceived for the cleft care and treatment outcomes.

\section{Conclusion}

In overall, nose is the most dissatisfied feature for both patients and parents. On the other hand, both parties showed the highest satisfaction towards hearing. Meanwhile, features related to facial appearance (nose, teeth, lip and facial profiles) requires more attention during cleft management.

\section{Acknowledgements}

We would like to thank the Director General of Health Malaysia for his permission to publish this paper. We are grateful to Dr. Siti Noor Fazliah Mohd Noor from School of Dental Sciences Universiti Sains Malaysia for her kindness and permission to use the questionnaires and CEP evaluation form.

\section{References}

Ali, S. Y., Abdul, Z. A., Mirani, S. A., Shaikh, M. I., Khattak, M. N., Sahito M. A. et al. (2015). Demographic data on the characterization of oral clefts in malaysia. Pakistan Oral \& Dental Journal, 35(1), 108-110.

Altman, D. G. (1999). Practical statistics for medical research. London: Chapman and Hall/ CRC, 403407.

Gatti, G. L., Freda, N., Giacomina, A., Montemagni, M., Sisti, A. (2017). Cleft lip and palate repair: our 
experience. The Journal of Craniofacial Surgery, $00(00), 1-7$.

Joycelyn, L. J., Penko, M. A., Rode, H.L. (1996). Cognition, communication, and hearing in young children with cleft lip and palate and in control children: a longitudinal study. Journal of Oral Maxillofacial Surgery, 97, 529-534.

Lansdown, R., Lloyd, J., Hunter, J. (1991). Facial deformity in childhood: severity and psychological adjustment. Child: care health and development, 17(3), 165-71.

Luyten, A., Evelien, D., Budolfsen, D., Hodges, A., Galiwango, G., Vermeersch, H. et al. (2013). Parental satisfaction in ugandan children with cleft lip and palate following synchronous lip and palatal repair. Journal of Communication Disorders, 46(4), 321-329.

Natsume, N., Kawai, T. (1986). Incidence of cleft lip and cleft palate in 39, 696 japanese babies born during 1983. International Journal of Oral Maxillofacial Surgery,15(5), 565-568.

Noar, J. H. (1991). Questionnnaire survey of attitudes and concerns of patients with cleft lip and palate and their parents. Cleft Palate-Craniofacial Journal, 28(3), 279-284.

Noor, S., Musa, S. (2007) Assessment of patients' level of satisfaction with cleft treatment using the cleft evaluation profile. Cleft Palate-Craniofacial Journal, 44(3), 292-303.

Oral Health Division, Ministry of Health Malaysia. (2009). National Oral Health Survey of School
Children (NOHSS) 2007 6-Year-Olds. Oral Health Division, Ministry of Health Malaysia. 38-39.

Oosterkamp, B. C. M., Dijkstra, P. U., Remmelink, H. J., Van, Oort, R. P., Goorhuis-Brouwer, S. M., Sandham, A. et al. (2007). Satisfaction with treatment outcome in bilateral cleft lip and palate patients. International Journal of Oral Maxillofacial Surgery, 36(10), 890-895.

P., Ha, C., Li, B., Shi. (2016). Parent satisfaction with primary repair of paediatric cleft lip in southwest china. International Association of Oral and Maxillofacial Surgeons, 5-9.

Sell, D., Mildinhall, S., Albery, L., Wills, A. K., Sandy, J. R., Ness, A. R. et al. (2015). The Cleft Care uk study. Part 4: perceptual speech outcomes. Orthodontics and Craniofacial Research, 18(2), 36-46.

Turner, S. R., Thomas, P. W. N., Dowell, T., Rumsey, N., Sandy, J. R. (1997). Psychological outcomes amongst cleft patients and their families. British Journal of Plastic Surgery, 50, 1-9.

Williams, Alison, C., David, B., Sue, M., Terrie, M., Debbie, S. et al. (2001). Cleft lip and palate care in the United Kingdom - The clinical standards advisory group (CSAG) Study. Part 2: Dentofacial outcomes and patient satisfaction. Cleft PalateCraniofacial Journal, 38(1), 24-29. 